\title{
Prognostic Factors for Postsurgical Recovery of Deltoid Palsy due to Cervical Disc Herniations
}

\author{
Jae-Yoon Chung ${ }^{1}$, Jong-Beom Park ${ }^{1,2}$, Han Chang ${ }^{1}$, Kyung-Jin Song ${ }^{1}$, Jin-Hyok Kim ${ }^{1}$, \\ Chang-Hwa Hong ${ }^{1}$, Jung Sub Lee ${ }^{1}$, Sang-Hun Lee ${ }^{1}$, Kwang-Sup Song ${ }^{1}$, \\ Jae Jun Yang ${ }^{1}$, Jae-Hyung $\mathrm{Uh}^{1}$, Young-Tae Kim ${ }^{1}$, Jae Min Lee ${ }^{2}$ \\ ${ }^{1}$ Cervical Spine Study Group, Korean Society of Spine Surgery, Seoul, Korea \\ ${ }^{2}$ Department of Orthopedic Surgery, Uijeongbu St. Mary's Hospital, College of Medicine, The Catholic University of Korea, Uijeongbu, Korea
}

Study Design: Retrospective multicenter study.

Purpose: We aimed to investigate prognostic factors affecting postsurgical recovery of deltoid palsy due to cervical disc herniation (CDH).

Overview of Literature: Little information is available about prognostic factors affecting postsurgical recovery of deltoid palsy due to $\mathrm{CDH}$.

Methods: Sixty-one patients with CDH causing deltoid palsy (less than grade 3) were included in this study: 35 soft discs and 26 hard discs. Average duration of preoperative deltoid palsy was 11.9 weeks. Thirty-two patients underwent single-level surgery, 22 two-level, four three-level, and three four-level. Patients with accompanying myelopathy, shoulder diseases, or peripheral neuropathy were excluded from the study.

Results: Deltoid palsy (2.4 grades vs. 4.5 grades, $p<0.001$ ) and radiculopathy (6.4 points vs. 2.1 points, $p<0.001$ ) significantly improved after surgery. Thirty-six of 61 patients (59\%) achieved full recovery (grade 5) of deltoid palsy, with an average time of 8.4 weeks. Longer duration of preoperative deltoid palsy and more severe radiculopathy negatively affected the degree of improvement in deltoid palsy. Age, gender, number of surgery level, and disc type did not affect the degree of improvement of deltoid palsy. Contrary to our expectations, severity of preoperative deltoid palsy did not affect the degree of improvement. Due to the shorter duration of preoperative deltoid palsy, in the context of rapid referral, early surgical decompression resulted in significant recovery of more severe grades (grade 0 or 1 ) of deltoid palsy compared to grade 2 or 3 deltoid palsy.

Conclusions: Early surgical decompression significantly improved deltoid palsy caused by $\mathrm{CDH}$, irrespective of age, gender, number of surgery level, and disc type. However, longer duration of deltoid palsy and more severe intensity of preoperative radiating pain were associated with less improvement of deltoid palsy postoperatively.

Keywords: Prognostic factors; Postsurgical recovery; Deltoid palsy; Cervical disc herniation

\section{Introduction}

Operative indications for cervical disc herniation $(\mathrm{CDH})$ are progressive or severe neurologic deficits or intractable radiating pain not responding to conservative treatment. The surgery for $\mathrm{CDH}$ is intended to relieve radiating pain

Received Feb 3, 2015; Revised Feb 14, 2015; Accepted Feb 14, 2015

Corresponding author: Jong-Beom Park

Department of Orthopedic Surgery, Uijeongbu St. Mary's Hospital, College of Medicine,

The Catholic University of Korea, 271 Cheonbo-ro, Uijeongbu 11765, Korea

Tel: +82-31-820-3578, Fax:+82-31-847-3671, E-mail: spinepjb@catholic.ac.kr 
Table 1. Muscle grading based on the manual motor power test

\begin{tabular}{ll}
$\begin{array}{ll}\text { Muscle grade } \\
5 \text { Normal }\end{array}$ & Complete range of motion against gravity with full resistance \\
\hline 4 Good & Complete range of motion against gravity with some resistance \\
3 Fair & Complete range of motion against gravity \\
2 Poor & Complete range of motion with gravity eliminated \\
1 Trace & Evidence of slight contractibility; no joint motion \\
\hline 0 Zero & No evidence of contractibility \\
\hline
\end{tabular}

and to relieve motor or sensory loss [1,2]. CDH is approached anteriorly or posteriorly. The surgical outcomes of $\mathrm{CDH}$ have been reported to be successful in terms of clinical and radiological measures $[3,4]$. To date, however, little information has been available on the surgical outcomes of deltoid palsy due to $\mathrm{CDH}$, because there are relatively few cases of deltoid palsy due to $\mathrm{CDH}$.

Our literature review yielded only one study that reported surgical outcomes of deltoid palsy due to $\mathrm{CDH}$ [5]. Chang et al. [5] reported that surgical decompression significantly improves the degree of deltoid palsy due to cervical radiculopathy. They also reported that deltoid palsy can occur in patients with $\mathrm{CDH}$ at C3-4 and C5-6, as well as $\mathrm{CDH}$ at $\mathrm{C} 4-5$, due to variations in motor distribution or anomalies of the cervical nerve roots. However, there are two limitations to Chang et al. [5]' study. The first is the small number of cases (14 cases) included in their study. The second is the absence of an analysis of prognostic factors affecting recovery of deltoid palsy due to $\mathrm{CDH}$, after surgery. Therefore, we performed this retrospective multicenter study on the largest series of $\mathrm{CDH}$ cases associated with deltoid palsy, in the literature to date, in order to investigate prognostic factors affecting postsurgical recovery of deltoid palsy due to $\mathrm{CDH}$.

\section{Materials and Methods}

This retrospective multicenter study was approved by the Institutional Review Boards of the 13 participating hospitals that provided the data. We included a total of 61 patients who underwent surgery between 1998 and 2013 for $\mathrm{CDH}$-related deltoid palsy that did not respond to conservative treatment. All patients complained of radiating pain to the scapula, shoulder, or arm, with weakness of shoulder abduction. The severity of deltoid palsy was classified into five grades, according to the manual motor power test described by Klein and Garfin (Table 1) [6]. Neurologic examination showed weakness of shoulder abduction (less than grade 3) due to deltoid palsy, in all patients. However, there were no pathologic reflexes, long tract signs, or increased deep tendon reflexes in any of the patients. The severity of radiculopathy was recorded on a visual analog scale with scores from 0 to 10 points. The type of disc (soft disc or hard disc) was determined based on plain radiography, computerized tomography scans, magnetic resonance images, and operative findings. Patients with accompanying myelopathy, shoulder diseases, or peripheral neuropathy were excluded from the study. We also excluded patients with grade 4 weakness of shoulder abduction due to deltoid palsy, to minimize inter-examiner variation in muscle power grading.

The average age of the 61 patients was 51.4 years (range, 25-69 years). There were 49 men and 12 women. Thirtyfive patients had $\mathrm{CDH}$ involving soft discs, and 26 patients had $\mathrm{CDH}$ involving hard discs. Thirty-two patients underwent single-level surgery (C4-5, 22; C5-6, 9; C3-4, 1), 22 had two-level surgery (C4-5-6, 19; C3-4-5, 2; C5-6-7, 1), four had three-level surgery $(\mathrm{C} 4-5-6-7,4)$, and three had four-level surgery $(\mathrm{C} 3-4-5-6-7,3)$. Anterior cervical discectomy and fusion was performed in 57 patients, artificial disc replacement in 2 patients, and foraminotomy/discectomy in 2 patients. Average follow-up was 20.7 months (range, 6-83 months). Follow-up measurements were taken before surgery and postoperatively at 1, 3, 6, and 12 months, as well as at last follow-up.

The Wilcoxon signed rank test was used to evaluate the degree of improvement in the severity of deltoid palsy and radiculopathy following surgery. In addition, the Pearson test and $t$-test were used to investigate prognostic factors of improvement in deltoid palsy after surgery. A $p$-value less than 0.05 was considered statistically significant. 
Table 2. Improvement of severity of deltoid palsy and radiculopathy after surgery

\begin{tabular}{lccc} 
Variable & Preoperative & Last follow-up & $p$-value \\
Deltoid palsy (grade) & $2.4 \pm 0.7$ & $4.5 \pm 0.7$ & $<0.001$ \\
Radiculopathy (VAS) & $6.4 \pm 1.9$ & $2.1 \pm 1.3$ & $<0.001$ \\
\hline
\end{tabular}

VAS, visual analog scale.

${ }^{a)} p$-value by the Wilcoxon signed rank test.

Table 3. Prognostic factors affecting the degree of improvement in deltoid palsy after surgery

\begin{tabular}{lcc} 
Factor & Correlation coefficient & $p$-value \\
\hline Duration of preoperative deltoid palsy & -0.350 & $<0.001$ \\
Severity of radiculopathy (arm VAS) & -0.338 & $<0.001$ \\
\hline Age & -0.170 & 0.19 \\
No. of surgery levels & -0.206 & 0.11 \\
Severity of preoperative deltoid palsy & 0.057 & 0.66
\end{tabular}

VAS, visual analog scale.

a)-value by the Pearson test.

\section{Results}

The mean duration of deltoid paralysis was 11.9 weeks (range, 1-156 weeks). Deltoid palsy was improved an average of 2.1 grades after surgery (grade $2.4 \pm 0.7$ vs. grade $4.5 \pm 0.7, p<0.001)$. Radiculopathy was also improved at an average of 4.3 points after surgery ( $6.4 \pm 1.9$ vs. $2.1 \pm 1.3$, $p<0.001$ ) (Table 2). Thirty-six of 61 patients $(59 \%)$ achieved full recovery (grade 5) of deltoid palsy, with an average time of 8.4 weeks (range, 1-54 weeks).

The results of the Pearson test are shown in Table 3. Longer duration of preoperative deltoid palsy negatively affected the degree of improvement in deltoid palsy after surgery. The degree of improvement in deltoid palsy was significantly lower in patients with a duration of deltoid palsy $>3$ months, compared to patients with duration of deltoid palsy $\leq 3$ months $(1.3 \pm 0.7$ grades vs. $2.2 \pm 0.9$ grades, $p<0.01$ ). Severe radiculopathy (arm visual analog scale [VAS]) negatively affected the degree of improvement of deltoid palsy after surgery. The degree of improvement in deltoid palsy was significantly lower in patients with arm VAS $>5$ points, compared to patients with arm VAS $\leq 5$ points $(1.9 \pm 0.9$ grades vs. $2.5 \pm 0.8$ grades, $p<0.05)$. However, neither age nor the number of surgery levels affected the degree of improvement in deltoid palsy after surgery. Contrary to our expectations, the severity of preoperative deltoid palsy also did not affect the degree of improvement in deltoid palsy after surgery. In seven patients with more severe grades of deltoid palsy (grade 0 or 1), preoperative duration of deltoid palsy was quite shorter compared to the 54 patients with grade 2 or 3 (5.1 weeks vs. 12.8 weeks, $p<0.05$ ). Due to the shorter duration of preoperative deltoid palsy, in the context of rapid referral, significant recovery was possible in more severe grades (grade 0 or 1 ) of deltoid palsy after surgery, compared to grade 2 or 3 deltoid palsy ( $4.0 \pm 0.0$ grades vs. $1.8 \pm 0.7$ grades, $p<0.001$ ).

The degree of deltoid palsy improvement was not statistically different between soft disc and hard disc (2.0 \pm 0.9 grades vs. $2.1 \pm 1.0$ grades, $p=0.556$ ). The degree of deltoid palsy improvement was also not statistically different between men and women (2.0 \pm 1.0 grades vs. $2.4 \pm 0.9$ grades, $p=0.173$ ).

\section{Discussion}

In this study, surgical decompression significantly improved the degree of deltoid palsy and radiculopathy due to $\mathrm{CDH}$. Longer duration of deltoid palsy preoperatively was associated with less improvement in deltoid palsy postoperatively. More severe intensity of radiating pain also adversely affected the degree of improvement in deltoid palsy. However, age, gender, number of surgery levels, and disc type did not affect the degree of improvement in 
deltoid palsy. Contrary to our expectations, the severity of preoperative deltoid palsy did not affect the degree of improvement in deltoid palsy. Due to the shorter duration of preoperative deltoid palsy, in the context of rapid referral, early surgical decompression resulted in significant recovery of more severe grades (grade 0 or 1 ) of deltoid palsy. Thus, we recommend early surgical decompression for better recovery of deltoid palsy in patients with $\mathrm{CDH}$ and associated deltoid palsy.

Due to the relatively small number of cases of $\mathrm{CDH}$ associated with deltoid palsy, the surgical outcomes of deltoid palsy due to $\mathrm{CDH}$ have not been fully described in the literature. Only one previous study by Chang et al. [5] reported that surgical decompression significantly improves the degree of deltoid palsy. However, they did not analyze prognostic factors affecting the recovery of deltoid palsy due to $\mathrm{CDH}$ after surgery. They therefore concluded that a relatively short duration of deltoid palsy might be attributed to satisfactory results of surgical outcomes. Our findings demonstrate that a longer preoperative duration of deltoid palsy is associated with less improvement in deltoid palsy postoperatively. A deeper analysis of our findings showed that when the preoperative duration of deltoid palsy was $>3$ months, there was a lower degree of improvement in deltoid palsy, compared to when the preoperative duration of deltoid palsy was $\leq 3$ months (1.3 grades vs. 2.2 grades, $p<0.01$ ). This result indicates that the preoperative duration of deltoid palsy significantly affects the degree of recovery of deltoid palsy after surgery. Our results also demonstrated an adverse effect of the intensity of radiating pain on recovery of deltoid palsy. When preoperative radiating pain was $>$ VAS 5 points, there was less improvement in deltoid palsy, compared to when the preoperative VAS was $\leq 5$ points (grade 1.9 vs. grade $2.5, p<0.05$ ). These results indicate that early surgical decompression should be recommended for better recovery of deltoid palsy after surgery in patients with $\mathrm{CDH}$ and associated deltoid palsy.

Age and gender were not found to affect the degree of improvement in deltoid palsy after surgery in our series. Deltoid palsy can occur in patients with $\mathrm{CDH}$ at C3-4 and C5-6, as well as $\mathrm{CDH}$ at $\mathrm{C} 4-5$, due to variations in motor distribution or anomalies of the cervical nerve roots [7-11]. In our series, 32 patients underwent single-level surgery, 22 two-level surgery, four three-level surgery, and three four-level surgery. In the 29 patients who underwent multi-level surgery, C4-5 was included in the extent of the surgery, which resulted in significant improvement in deltoid palsy. The type of disc (soft disc or hard disc) had no significant effect on the degree of recovery of deltoid palsy. Discectomy with sufficient decompression of uncovertebral joints is seen as a key factor for satisfactory recovery of deltoid palsy, in severe hard disc (spondylotic radiculopathy) with accompanying deltoid palsy. Contrary to our expectations, the severity of preoperative deltoid palsy did not affect the degree of improvement in deltoid palsy after surgery. Several factors may account for this unexpected result. In the seven patients with more severe grades of deltoid palsy (grade 0 or 1), preoperative duration of deltoid palsy was shorter than in the 54 patients with grade 2 or 3 deltoid palsy (5.1 weeks vs. 12.8 weeks, $p<0.05$ ). Due to rapid referral, early surgical decompression was possible. Therefore, there was a significantly greater degree of recovery of deltoid palsy in patients with more severe grades (grade 0 or 1 ) than patients with grade 2 or 3 deltoid palsy ( 4.0 grades vs. 1.8 grades, $p<0.001)$. Again, these results emphasize the importance of early surgical decompression, rather than conservative treatment, in patients with $\mathrm{CDH}$ and associated with deltoid palsy.

However, there are some limitations in this study. First, this study is retrospective study on a large number of centers with all the potential inaccuracies that implies. Second, electrophysiological rather than clinical assessment would be a more objective criteria for serial evaluation of muscle strength recovery.

\section{Conclusions}

We found that surgical decompression significantly improved deltoid palsy caused by $\mathrm{CDH}$ irrespective of age, gender, number of surgery levels, and disc type. However, the longer duration of deltoid palsy and more severe intensity of preoperative radiating pain were associated with less improvement in deltoid palsy postoperatively. Thus, early surgical decompression is recommended for better recovery of deltoid palsy in patients with $\mathrm{CDH}$ and associated deltoid palsy.

\section{Conflict of Interest}

No potential conflict of interest relevant to this article was reported. 


\section{References}

1. Caridi JM, Pumberger M, Hughes AP. Cervical radiculopathy: a review. HSS J 2011;7:265-72.

2. Gebremariam L, Koes BW, Peul WC, Huisstede BM. Evaluation of treatment effectiveness for the herniated cervical disc: a systematic review. Spine (Phila Pa 1976) 2012;37:E109-18.

3. Decker RC. Surgical treatment and outcomes of cervical radiculopathy. Phys Med Rehabil Clin N Am 2011;22:179-91.

4. Smith JS, Helgeson MD, Albert TJ. The argument for anterior cervical discectomy and fusion over total disk replacement. Semin Spine Surg 2012;24:2-7.

5. Chang H, Park JB, Hwang JY, Song KJ. Clinical analysis of cervical radiculopathy causing deltoid paralysis. Eur Spine J 2003;12:517-21.

6. Klein JD, Garfin SR. Clinical evaluation of patients with suspected spine problems. In: Frymoyer JW, Ducker TB, Hadler NM, Kostuik JP, Weinstein JN, Whitecloud TS, editors. The adult spine: principles and practice. Philadelphia, PA: Lippincott-Raven; 1997. p.319-40.

7. Kubo Y, Waga S, Kojima T, Matsubara T, Kuga Y, Nakagawa Y. Microsurgical anatomy of the lower cervical spine and cord. Neurosurgery 1994;34:895-90.

8. Marzo JM, Simmons EH, Kallen F. Intradural connections between adjacent cervical spinal roots. Spine (Phila Pa 1976) 1987;12:964-8.

9. Moriishi J, Otani K, Tanaka K, Inoue S. The intersegmental anastomoses between spinal nerve roots. Anat Rec 1989;224:110-6.

10. Shimizu S, Tachibana S, Sagiuchi T, Kurita M, Fujii K. Radiculopathy at the C5/6 intervertebral foramen resulting in isolated atrophy of the deltoid: an aberrant innervation complicating diagnosis: report of two cases. Eur Spine J 2008;17 Suppl 2:S338-41.

11. Shinomiya K, Okawa A, Nakao K, et al. Morphology of $\mathrm{C} 5$ ventral nerve rootlets as part of dissociated motor loss of deltoid muscle. Spine (Phila Pa 1976) 1994;19:2501-4. 